\title{
Problemas médico-legales del consumo de drogas
}

\author{
Serrat Moré, D. \\ Prof ${ }^{a}$ Titular de Medicina Legal Universidad de Zaragoza
}

Enviar correspondencia:

Dolores Serrat Moré. Facultad de Medicina. Universidad de Zaragoza. C/ Domingo Miral s/n. 50009 Zaragoza. e-mail: dserrat@posta.unizar.es o bien dserratm@aeds.org

\section{RESUMEN}

Los trastornos derivados del consumo de alcohol y drogas son causa de múltiples problemas en diferentes ámbitos del Derecho, siendo necesario para su resolución que Jueces y Tribunales dispongan de un informe pericial que valore el estado del paciente y que les permita conocer la situación real, para acorde con ello dictar una resolución motivada.

Para la valoración psicopatológica proponemos atender a 2 criterios: uno médico basado en los síntomas y signos clínicos, la gravedad de cuadro y el momento de inicio del mismo en relación con los hechos de trascendencia jurídica, y otro médico legal, que relaciona los hechos con el estado del paciente partiendo de un análisis estructural de sus funciones cognitivas y volitivas y del estudio de la gravedad de la adicción.

Dada la frecuencia con la que las conductas adictivas coexisten con otras patologías psiquiátricas, proponemos un estudio completo recurriendo a métodos prospectivos (seguimiento del paciente), retrospectivos (basado en autoinformes referidos a periodos de tiempo concretos) y objetivos (análisis y distintas pruebas de laboratorio) para el correcto diagnóstico diferencial entre los trastornos inducidos por drogas y otros trastornos psiquiátricos.

Para la valoración pronostica, proponemos estudiar los elementos que pueden influir en el éxito o el fracaso de la asistencia médica o psicosocial del problema, atendiendo básicamente a: 1) los factores que motivan el consumo, 2) los motivos que motivan la solicitud de ayuda para abandonar el consumo, y 3) viabilidad del plan Terapéutico propuesto.

Palabras clave: drogas, problemas legales, informe pericial, justicia, informe psiquiátrico, comorbilidad.

\section{SUMARY}

The dysfunctions derived from the consumption of alcohol and drugs are the cause of multiple problems in different spheres of Law. To resolve them, judges and tribunals require an expert report assessing the condition of the patient enabling them to ascertain the real situation prior to reaching a decision.

For the psychopathological evaluation we propose 2 criteria, one medical based on the clinical symptoms and signs, the seriousness of the case and the commencement of the same in relation to the facts of the legal case in question, and another legal- medical one which lists the facts with the medical condition of the patient on the basis of a structural analysis of his/her cognitive and volitive functions and a study of the seriousness of the addiction.

Given the frequency with which addictive behaviours coexist with other psychiatric pathologies, we propose a complete study based on prospective methods (patient followups), retrospectives (based on self-reports referring to specific periods) and objectives (analysis and different laboratory tests) for the correct differential diagnosis between dysfunctions induced by drugs and other psychiatric dysfunctions.

Lastly and for the evaluation for the prognosis, we propose to study the elements that can influence the success or the failure of the medical or psychosocial attention to the problem, basically: 1) the factors that motivate the consumption, 2) the reasons that motivate the request for assistance in giving up consumption, and 3) viability of the proposed therapeutic plan.

Key words: drugs, legal problems, expert report, justice, psychiatric report, comorbility.

\section{INTRODUCCIÓN}

$\mathbf{L}$ as drogas, entendidas en un concepto amplio como sustancias naturales o sintéticas que producen una alteración de la conducta y/o del estado psíquico que inducen a repetir el consumo y al uso continuado (OMS), se incluyen en las actuales clasificaciones de las enfermedades mentales como causa de trastornos que van desde la intoxicación aguda no complicada y el consumo perjudicial, hasta los cuadros psicóticos y la demencia manifiesta (Rodes y Martí, 1997). Así la CIE-10 los denomina "Trastornos 
mentales y del comportamiento debidos al consumo de sustancias psicotropas" y la DSM-IV "Trastornos relacionados con sustancias".

Para la OMS, la drogodependencia es " un estado consecuente a la administración repetida de una droga de forma periódica o continua, que varía según el agente utilizado", constituyendo sin lugar a dudas una de las plagas de nuestro tiempo que plantea a la vez un grave problema sanitario, por las consecuencias perjudiciales para la salud, un problema social, moral y legal al ser una de las causas directamente relacionadas con la marginación y la delincuencia.

Desde el punto de vista sanitario se considera la drogadicción como un problema de salud pública, al suponer un riesgo real para la salud tanto individual como colectivo, y por tanto un desequilibrio entre el mejor estado de salud de la población de un país, los riesgos que afecten a la salud de cada individuo o de la población, y los medios existentes en la colectividad para controlar estos riesgos. Si bien durante un tiempo se puso en tela de juicio si la adicción a drogas era una enfermedad o simplemente una conducta antinormativa, parece que hoy no existen y se califica como una enfermedad que causa no solamente una pérdida del estado psicofísico de salud, sino que provoca un cambio de conducta y de costumbres en los sujetos, que se ha puesto en relación con las modificaciones cuantitativas y cualitativas que se insertan en los sustratos biológicos de la personalidad.

Desde el punto de vista social y moral drogarse se ha considerado (San Martín, 1982) como una infracción calificable como delictiva, pues en si constituye una forma de autoviolencia que en el limite es suicida. El daño a uno mismo y el suicidio han sido contemplados siempre de alguna manera como hechos ilícitos, como una clara agresión a la integridad del ciudadano, considerándose que en la autointoxicación crónica se transgrede el inderogable deber de solidaridad social, la indeclinable obligación de desarrollar, según las posibilidades de cada cual, una actividad tendente al progreso material y espiritual de la sociedad, y quebrantándose el cumplimiento de las obligaciones cívicas por cuanto se traslada a la colectividad el deber de cargar con unos costes sanitarios, laborales y daños de todo tipo (familiares, de seguridad publica etc). En este sentido el hábito de drogarse no puede entenderse como el ejercicio de la libertad personal sino como una especie de "delito ecológico" contra el propio cuerpo y en consecuencia el contaminarse a si mismo y perturbar secundariamente a los demás es algo que probablemente debe ser calificado en esencia como antisolidario y antidemocrático.

Legalmente el consumo de drogas no es considerado en sí mismo como un hecho delictivo, sin embargo hasta fechas relativamente próximas a los drogadictos se les ha considerado como "sujetos peli- grosos", y aún hoy a las drogas se las considera como un factor criminógeno de primer orden.

Por todo ello consideramos que un estudio de las consecuencias legales del consumo de drogas es de gran interés para todos aquellos profesionales que en un momento determinado puedan ser requeridos para emitir un informe pericial en dicha materia, y entendemos que para que éste cumpla con los criterios de calidad exigibles se requieren por parte del perito conocimientos específicos en materia de drogas, conocimiento del método pericial y conocimientos básicos de derecho, pues como dice Piga "hay que conocer el "porqué" para dirigir el "como" de nuestras actuaciones".

Atendiendo pues a estas exigencias distinguiremos en este trabajo 3 apartados:

1. Conceptos básicos en relación con el consumo de sustancias.

2. Aspectos básicos del tratamiento legal de las drogas.

3. El informe pericial en materia de drogas.

\section{Conceptos básicos en relación con el consumo de sustancias.}

Desde el punto de vista médico legal nos interesa distinguir los trastornos relacionados con las sustancias en dos grupos:

I. Los trastornos por el consumo de sustancias: el abuso y la dependencia.

II. Los trastornos inducidos por las sustancias:

-Específicos: Intoxicación y abstinencia

-Otros, que también pueden ser inducidos por otras causas orgánicas, que se corresponden con lo que tradicionalmente se han denominado trastornos psicoorgánicos o trastorno mental orgánico, y que pueden ser cuadros de delirium, demencia, trastornos mnésicos, etc.

De acuerdo con esta clasificación y las referencias legales a estos trastornos consideramos de gran interés la definición de los siguientes términos: abuso, dependencia, adicción, intoxicación, y abstinencia.

Se entiende por abuso la situación de consumo continuado de una o varias sustancias que se mantiene a pesar de que el mismo tenga consecuencias negativas para el sujeto y estén afectando a su salud, física o mental. Equivale a lo que la CIE-10 denomina consumo perjudicial.

Los criterios para su clasificación según la DSM-IV requieren, que este patrón desadaptativo se mantenga durante al menos 12 meses, y de lugar a uno o más de los siguientes ítems: incumplimiento de obligaciones importantes en el trabajo, casa, etc; realización de actividades físicamente peligrosas bajo sus 
efectos; problemas legales relacionados con la sustancia; o problemas sociales o interpersonales derivados de sus efectos; y todo ello sin que se cumplan los criterios de dependencia.

La dependencia se define como un conjunto de síntomas cognoscitivos, comportamentales o fisiológicos que indican que el individuo ha perdido el control de uso de una sustancia psicoactiva y que la continúa consumiendo a pesar de la aparición de problemas significativos relacionados con ella.

El patrón desadaptativo de uso se caracteriza por la tolerancia, que es la necesidad de aumentar progresivamente la dosis para obtener los efectos; la abstinencia; la ingestión compulsiva de la sustancia, y la necesidad irresistible de consumo (Carrasco y Maza, 1996). Custer (1986) nos dice que la dependencia clínica sólo es identificable con la dependencia psicosocial (determinante de la adicción) cuando concurren los componentes conductuales básicos que son: preocupación por la droga, el uso compulsivo y la tendencia a la recaída.

La adicción se identifica con un estado o situación, y es considerado como una respuesta a una exposición prolongada a una droga. Se caracteriza por una tendencia hacia un consumo progresivamente mayor, y una disposición persistente a volver al uso de la droga cuando se consigue la abstinencia y se ha superado la dependencia fisica.

Íntimamente ligado con los conceptos de dependencia (muchas veces utilizado como sinónimo de adicción) y de adicción se encuentra el concepto de tolerancia que puede definirse siguiendo a Delgado (1998) como la pérdida del efecto de la droga por el uso constante de la misma, lo que crea la necesidad de incrementar la dosis para obtener el mismo efecto, y la suspensión del consumo provoca la abstinencia.

Por otra parte el fenómeno de la tolerancia no es ilimitado sino que se ha observado que existe un techo de la tolerancia, es decir un nivel máximo a partir del cual no se producen nuevos incrementos de la dosis, considerándose que este nivel en relación con los opiáceos es aproximadamente de 10 veces la dosis original.

La intoxicación por sustancias se define en el DSM IV como la aparición de un síndrome reversible específico de cada sustancia tras la reciente ingestión de la misma, y lleva consigo la presencia de cambios psicológicos o comportamentales desadaptativos, clínicamente significativos, debido al efecto de la sustancia sobre el sistema nerviosos central, que se presenta durante el consumo de la sustancia o poco tiempo después.

Es por tanto un estado transitorio, de intensidad variable según la fase metabólica, el tipo de sustancia, la dosis y la propia individualidad del sujeto, siendo bien conocido que no todos los organismos responde igual a la administración de la droga sino que existen disposiciones personales de mayor riesgo toxicofílico.

El síndrome de abstinencia debe entenderse como el cuadro clínico que se configura tra el consumo reiterado de sustancias, generalmente prolongado o a dosis elevadas, y que se traduce en un conjunto de síntomas con diferente expresión, intensidad y gravedad, que aparecen en relación con una sustancia determinada y a la dosis consumida, trás una abstinencia en la ingesta o administración, absoluta o relativa en el consumo.

El cuadro de abstinencia es uno de los indicadores de la dependencia, y sirve para diferenciar ésta del abuso y del consumo perjudicial.

La expresión clínica del síndrome de abstinencia es variable, dependiendo esta variabilidad no sólo de la sustancia y la dosis, sino también de las respuestas individuales, e incluso de otros factores externos o estímulos que pueden contribuir a una situación de tensión y ansiedad sobreañadida, relacionados en general con la imposibilidad que tiene el dependiente de controlar y manejar el entorno y por tanto de conseguir la sustancia o un sustitutivo.

Para la valoración de la intensidad del síndrome de abstinencia Maurel y Voger (1967) propusieron una clasificación en cuatro grados: 1) Leve, caracterizado por bostezos, lagrimeo, rinorrea, estornudos, y sudoración; 2) Moderado, caracterizado además por anorexia, midriasis, temblor, piloerección aumento de la presión arterial; 3) Marcado, cuando se añade además fiebre, respiración profunda, inquietud e insomnio y 4) Severo, si además aparecen vómitos, diarrea y pérdida de peso. Y en el mismo sentido Christiani y Stübing (1977) propusieron un cuadro de gravedad de los síntomas de desintoxicación en 5 grados:

1) Grado 0, que se corresponde más que con un cuadro de abstinencia con un síndrome de querencia, es decir de necesidad de droga, angustia, nerviosismo y desorientación.

2) Grado 1, cuando aparecen los primeros síntomas, como bostezos, sudoración lagrimeo, rinorrea y modificaciones de la personalidad, que se corresponde con el estado a las 4-8 horas.

3) Grado 2, que se corresponde con la agudización de los síntomas anteriormente mencionados, midriasis, piloerección, calambres musculares, temblores, acaloramiento, anorexia y dolores musculares y óseos, correspondiéndose con el estado a las 12 horas.

4) Grado 3, cuando además de agudizarse los síntomas anteriores aparece el insomnio, el aumento de la presión arterial y de la temperatura, la respiración se hace rápida y profunda, hay taquicardia y náuseas, lo que suele manifestarse a las 18-24 horas.

5) Grado 4, evidente sobre las 24-36 horas de la abstinencia, y que se caracteriza porque junto a todo 
lo anterior se aprecia enrojecimiento facial, vómitos, diarrea y pérdida de peso.

\section{Aspectos básicos del tratamiento legal de las drogas.}

Los problemas que plantean los trastornos relacionados con las drogas pueden tener incidencia en los distintos ámbitos del Derecho: Penal, Civil, Laboral y Administrativo, dando lugar a situaciones periciales variadas y que precisan conocimiento de la regulación legal que a continuación analizaremos.

\section{DROGASY DERECHO PENAL}

Es indudable que la consideración jurídico penal del drogadicto ha evolucionado mucho desde la antigua declaración como sujeto peligroso en la LPRS de 1970 a la actual consideración en las normas penales y la jurisprudencia, evolución en la que ha tenido mucho que ver el impulso que supuso la puesta en marcha en 1985 de un Plan Nacional sobre Drogas iniciado desde la convicción de que la lucha contra este fenómeno social creciente debía hacerse desde la prevención tanto individual como colectiva.

Desde el punto de vista legal la Ley Orgánica 1/1988 de reforma del Código Penal en materia de tráfico de drogas supuso un paso significación en el tratamiento jurídico-penal del drogodependiente al primar en sus disposiciones la orientación preventiva en las sanciones penales al disponer que la autoridad judicial puede conceder el beneficio de remisión condicional en el caso de que el reo se hubiera deshabituado o se encontrare en tratamiento para ello, y completa en el nuevo código penal al incluir como circunstancias modificadoras de la responsabilidad criminal diversas circunstancias psicopatológicas vinculadas al consumo de drogas.

El estudio de los problemas de las drogas en el ámbito del derecho penal los agruparemos en:

1. Las drogas como factor criminógeno

2. Las drogas como factor modificador de la responsabilidad criminal.

3. El tratamiento jurídico-penal de la drogadicción.

4. La intervención del perito.

\section{Las drogas como factor criminógeno}

La sistematización de los delitos relacionados con las drogas es algo complejo, que puede realizarse atendiendo a diversos criterios; así Merlo (1989) y De
Fazio (1990) nos aportan clasificaciones que permiten distinguir los delitos en 3 grupos:

1. Delitos directamente relacionados con la producción y distribución de la droga o criminalidad inducida, pues lo que supone es la violación de las leyes sobre tenencia y tráfico de sustancias. Se incluyen dentro de los delitos contra la Salud Pública y su gravedad penal viene determinada por diversas circunstancias entre las que destacan por su interés medico el tipo de sustancia (según cause o no graves daños a la salud), la cantidad de droga (notoria importancia) o que se trate de un conducta de extrema gravedad valorados todos los elementos objetivos y subjetivos del caso concreto enjuiciado.

2. Delitos cometidos por sujetos bajo la influencia y efectos del consumo de sustancias o criminalidad directa. Tienen interés médico legal pues es posible que modifiquen la imputabilidad, si se cumplen los criterios de los artículos 20.2 y 21.2 del Código Penal.

3. Delitos cometidos con el fin de procurarse el dinero necesario para mantener la dependencia, que podríamos denominar criminalidad indirecta. En este caso también su interés se relaciona con la posibilidad de merecer la consideración de eximente o atenuante de la responsabilidad criminal de acuerdo con los artículos 20.2 y 21.2 del Código Penal.

Por otra parte debemos recordar que la peligrosidad y criminalidad del drogadicto está íntimanente relacionada con el tipo de droga consumida y la fase de la adicción.

Atendiendo al tipo de droga, diremos que la peligrosidad y criminalidad esta relacionada con los efectos clínicos de la sustancia y a modo de ejemplo distinguimos entre la delitología del heroinómano, y la del cocainómano, y así aunque resulta evidente la asociación entre criminalidad y heroinomanía no es porque la heroína aumente la capacidad delictógena, sino porque la necesidad de obtenerla le obliga a delinquir. Por ello los delitos son más frecuentes en la fase de dependencia o abstinencia que en la fase de intoxicación. Por el contrario los cocainómanos delinquen durante la fase de intoxicación con más frecuencia que en la deprivación, siendo su conducta violenta muy variable, oscilando desde la agresión física hasta la violación o el homicidio.

Atendiendo a las fases de la adicción, distinguiremos entre la fase de dependencia, la fase de intoxicación y la fase de abstinencia.

En la fase de dependencia, y de forma bastante similar en casi todas la toxicomanías, la delincuencia

Delgado., S y cols: Psiquiatría Legal y Forense. Edit. Colex. Madrid, 1994. 
se relaciona con la imperiosa necesidad que tiene el sujeto de conseguir la droga, lo que le obliga a conseguirla a través de medios ilícitos (fraudes, robos etc), a relacionarse con profesionales de la delincuencia que requieren su colaboración y complicidad en sus delitos, y además se ha producido un decaimiento ético de la personalidad que facilita cualquier tipo de acción, incluso delictiva, sobre todo si existen obstáculos a la satisfacción de su hábito.

En la fase de intoxicación, la delitología está muy condicionada por los efectos farmacológicos de las sustancias consumidas, por ello en general se dice que las sustancias excitantes (cocaína, marihuana, anfetaminas) son más peligrosas que las depresoras (opiáceos), pues mientras éstos suelen tener una conducta tranquila, los adictos a sustancias excitantes pueden tener un comportamiento violento y agresivo con el cuadro de delirio tóxico que muchas veces presentan. Los alucinógenos son peligrosos para el propio consumidor sobre todo en relación a las conductas suicidas.

En la fase de abstinencia, la peligrosidad se correlaciona directamente con la aparición de un síndrome de abstinencia. Aquellas sustancias que no lo provocan la privación brusca de la sustancia tienen unos efectos homologables a los de la fase de dependencia o necesidad

\section{Las drogas como factor modificador de la res- ponsabilidad criminal.}

La peritación psiquiátrica en relación con la imputabilidad, requiere que se determine si en el momento de los hechos el delincuente se encontraba en alguna de las siguientes situaciones previstas en los artículos 20. 2 y 21.2 del Código Penal.

El art. 20. 2 del C.P. dice "Están exentos de responsabilidad criminal el que al tiempo de cometer la infracción penal se halle en estado de intoxicación plena por el consumo de bebidas alcohólicas, drogas tóxicas, estupefacientes, sustancias psicotrópicas u otras que produzcan efectos análogos, siempre que no haya sido buscado con el propósito de cometerla o no se hubiese previsto o debido prever su comisión, , o se halle bajo la influencia de un síndrome de abstinencia, a causa de su dependencia de tales sustancias, que le impida comprender la ilicitud del hecho o actuar conforme a esa comprensión."

Los requisitos necesarios para considerar la intoxicación por drogas como circunstancia eximente son:

1. Que la intoxicación sea plena, es decir exista una pérdida del propio dominio y de las facultades intelectivas.

2. Que el estado de intoxicación no haya sido buscado de propósito para cometer la infracción
3. Que la comisión de la infracción no se hubiera previsto o debido prever.

Es decir desde el punto de vista médico legal pueden incluirse en el concepto de intoxicación plena, la fortuita, es decir aquella no querida ni prevista pues el sujeto desconoce los efectos del tóxico, y la voluntaria, es decir aquella en la que se conocen los efectos de la droga y ésta se consume voluntariamente, aunque no se desea ni se prevén los conflictos judiciales, cuando alcanza la droga la máxima concentración en el organismo y por tanto su máxima alteración de funciones cognitivas y/o volitivas.

En relación con el síndrome de abstinencia, para que pueda considerarse como eximente es necesario que sea de tal intensidad que ocasione una abolición de la capacidad intelectiva que le impida comprender la ilicitud del hecho, o de la capacidad volitiva que le impida comportarse conforme a esa comprensión.

Cuando no se cumple el requisito de plenitud de la intoxicación o de intensidad del síndrome de abstinencia, y a tenor de lo dispuesto en el art. 21.1, serían eximentes incompletas y por tanto atenúan la responsabilidad criminal: "Las causas expresadas en el Capítulo anterior cuando no concurrieren todos los requisitos necesarios para eximir de responsabilidad en sus respectivos casos".

Por otra parte la prolongación del consumo de sustancias en el tiempo, que pudiera derivar en un cuadro de trastorno mental orgánico (delirium, demencia, etc.) daría lugar a una circunstancia eximente de responsabilidad criminal de acuerdo con lo dispuesto en el articulo 20.1 "El que al tiempo de cometer la infracción penal, a causa de cualquier anomalía o alteración psíquica, no pueda comprender la ilicitud del hecho o actuar conforme a esa comprensión".

Además y de acuerdo con el articulo 21.2, son circunstancias atenuantes: "la de actuar el culpable a causa de su grave adicción a las sustancias mencionadas en el número $2^{\circ}$ del articulo anterior".

Es quizá más difícil establecer el concepto de grave adicción, y en nuestra opinión sólo debería aplicarse cuando la grave adicción sea determinante del delito, aunque en el momento de su comisión no se encuentre el autor bajo los efectos de la sustancia, o en la situación de síndrome de abstinencia.

\section{El tratamiento jurídico-penal de la drogadicción.}

La nueva consideración jurídico penal del drogodependiente a partir de la reforma del Código Penal en materia de tráfico de drogas (LO1/1988 de 24 de marzo) y todo el desarrollo normativo posterior, el Código Penal de 1995, así como las propias estrategias contempladas en el Plan Nacional de Drogas, tanto el de 1985, como el actual documento de actualización y renovación (RD 1911/1999 de 17 de diciembre por el 
que se aprueba la Estrategia Nacional sobre drogas para el periodo 2000-2008) ponen de manifiesto que en el tratamiento de la criminalidad de los sujetos con toxicodependencias deben primar las actuaciones de carácter preventivo-asistencial sobre las penales, Io que en la práctica se traduce en la aplicación de las medidas de seguridad en sustitución o en concurrencia con la pena, y en la posibilidad que tiene el juez de aplicar la suspensión condicional de la pena.

\subsection{Aplicación de las medidas de seguridad.}

En nuestro Derecho Penal la aplicación de medidas de seguridad queda limitada a una serie de supuestos o presupuestos establecidos por la Ley y que son: 1) que el sujeto haya cometido un hecho previsto como delito, y 2) que del hecho y las circunstancias personales del sujeto pueda deducirse un pronóstico de comportamiento futuro que revele la probabilidad de comisión de nuevos delitos, en definitiva que pueda establecerse existencia de un estado de peligrosidad criminal; y además esta sometida al principio de proporcionalidad, es decir proporcional a la peligrosidad del sujeto y a la gravedad de los delitos que cabe esperar cometa en el futuro

Por otra parte, su aplicación esta sometida al principio de legalidad y por tanto sólo podrán aplicarse en los supuestos definidos por la Ley, y de acuerdo con Gracia, Boldova y Alastuey (1998) nuestro Código penal solo reconoce dos categorías de estados peligrosos: los inimputables y los semiimputables. Por otra parte, y como recuerda Romeo Casabona el juicio de peligrosidad requiere por una parte un diagnóstico de peligrosidad (es decir la comprobación de la cualidad de peligroso) y se basara en el estudio de las características del delito cometido, la personalidad del sujeto y la concurrencia de otros factores biológicos, ambientales, sociales, etc.; y de una prognosis criminal (comprobación de la relación entre dicha cualidad de peligroso y el futuro criminal del sujeto) que se fundamentara en un estudio de probabilidad que puede realizarse o bien siguiendo el método intuitivo (a partir de apreciaciones subjetivas del juez sin apoyo científico o técnico); el método científico (basado en el estudio de la personalidad del sujeto por especialistas mediante procedimientos científicos); o el método estadístico (se basa en la aplicación de tablas de predicción y el cálculo de probabilidades con la ayuda de estas tablas teniendo en cuenta los rasgos de personalidad del sujeto).

Dentro de las medidas de seguridad previstas en nuestro Código Penal (art.102.1) esta prevista la medida de internamiento en un centro de deshabituación para los que sean declarados exentos de responsabilidad penal por aplicación de la eximente de intoxicación plena o síndrome de abstinencia, o con responsabilidad atenuada por la aplicación de la eximente incompleta por los mismos motivos.
No dice nada el Código Penal respecto a la posibilidad de aplicar las medidas de seguridad a los declarados con responsabilidad penal atenuada por reconocimiento de una situación de grave adicción, es más si nos atenemos al tenor literal del articulo 104 del C.P. "En los supuestos de eximente incompleta en relación con los números $1^{\circ}, 2^{\circ}$ y $3^{\circ}$ del artículo 20, el juez o Tribunal podrá imponer, además de la pena correspondiente, las medidas previstas en los artículos...." debemos concluir que la aplicación de la medidas de seguridad es imposible en este caso. Pese a ello, el Tribunal Supremo ha interpretado recientemente el precepto estableciendo que sí es posible adoptar esas medidas de seguridad también en los casos en que no concurra eximente incompleta sino una mera atenuante (Sentencias de 11 de Abril de 2000, 20 de Octubre de 2000, 6 de marzo de 2001, 26 de octubre de 2001 y 27 de febrero de 2002).

\subsection{La aplicación de la suspensión condicional de la pena.}

El Código penal en su articulo 80 prevé la posibilidad de dejar en suspensión la ejecución de las penas privativas de libertad inferiores a dos años mediante una resolución motivada y atendiendo fundamentalmente a la peligrosidad criminal del sujeto, y en el articulo 81 establece como condiciones necesarias para dicha suspensión que el condenado haya delinquido por primera vez, que la pena impuesta no sea superior a 2 años de privación de libertad, y que se hayan satisfecho las responsabilidades civiles que se hubieren originado, salvo que el juez declare la imposibilidad total o parcial de que el condenado haga frente a las mismas.

Pero además de esto el articulo 87 del Código recoge que aún cuando no concurran las condiciones $1^{\mathrm{a}}$ y $2^{\mathrm{a}}$ del art. 81 (primer delito y pena no superior a 2 años) el Juez o Tribunal, cuando se hubiere cometido el hecho delictivo a causa de la dependencia al alcohol, drogas tóxicas, estupefacientes, sustancias psicotrópicas u otras de análogo naturaleza, podrá acordar la suspensión de la ejecución de las penas privativas de libertad no superiores a 3 años cuando se den las siguientes circunstancias: a) Que se certifique suficientemente por el centro o servicio público o privado debidamente acreditado u homologado que el condenado se encuentra deshabituado o sometido a tratamiento para tal fin; y 2) Que no se trate de reos habituales, entendiéndose como tales quienes hubieren cometido 3 o más delitos de los comprendidos en un mismo capítulo del Código Penal dentro de un periodo de tiempo no superior a cinco años $y$, además, hayan sido condenados por ello.

Además, en el caso de que sea reincidente para la decisión de conceder o no la suspensión condicional valorara su oportunidad o no atendidas las circunstancias del hecho y del autor, la suspensión de la ejecu- 
ción quedará siempre condicionada a que el reo no delinca en el periodo que se señale (de 3 a 5 años) y de que si esta sometido a tratamiento de deshabituación no lo abandone hasta su finalización, lo que lleva aparejada la obligación de los centro o servicios responsables de dicho tratamiento de facilitar al Juez o Tribunal la información precisa para comprobar el comienzo del a tratamiento, conocer periódicamente su evolución y las modificaciones que ha experimentado y su finalización.

Transcurrido el plazo de suspensión sin haber delinquido se acordara la suspensión de la pena si se ha acreditado la deshabituación o la continuidad del tratamiento del reo, de lo contrario se ordenara su cumplimiento, salvo que los informes emitidos por los especialistas estimen la continuación del tratamiento, en cuyo caso el juez podrá conceder razonadamente una prórroga del plazo de suspensión por un tiempo no superior a 2 años.

\section{Intervención del perito.}

A la vista de lo expuesto, las cuestiones que suelen plantearse al perito desde el punto de vista penal pueden concretarse en:

4.1. La valoración de la causalidad entre el delito cometido y el consumo de sustancias, y en concreto con el tipo de sustancia referida.

4.2. La valoración de la imputabilidad y sus posibles modificaciones habida cuenta que la drogadicción puede ser una circunstancia modificadora de la responsabilidad criminal, desde eximente (en los casos de intoxicación plena, síndrome de abstinencia, o cuando el consumo de sustancias es el responsable de una anomalía o alteración psíquica, siempre y cuando estos estados sean de suficiente gravedad como para anular la capacidad de comprender y actuar conforme a esta comprensión, y excepto que se hubieran consumido con la intención de delinquir), o atenuantes (bien por tratarse en los casos anteriores de situaciones de menor gravedad y que no anulan sino sólo disminuyen la capacidad de comprender y actuar conforme a esta comprensión, o bien de estados de grave adicción).

4.3. Las cuestiones relativas sobre la aplicación de las medidas de seguridad: tipo de medida, duración, etc.

4.4. Las cuestiones relativas a la aplicación de la suspensión condicional de la pena, su revocación o prórroga, etc.

\section{DROGASY DERECHO CIVIL}

Los problemas que el consumo de sustancias plantean en este ámbito del Derecho, se relacionan con las referencias explícitas a ellas que se hacen en el Código Civil y que afectan sobre todo a las relaciones de familia y al matrimonio, pero también con los internamientos involuntarios y en general con la capacidad civil.

El Código Civil en su artículo 82 regula las causas de separación matrimonial, y entre ellas en su apartado $4^{\circ}$, especifica que lo serán: el alcoholismo, la toxicomanía o las perturbaciones mentales, siempre que en interés del otro cónyuge o el de la familia exijan la suspensión de la convivencia

Por otra parte no existe ninguna duda sobre que en algunos casos el abuso, dependencia u otros trastornos inducidos por sustancias, pueden considerarse como enfermedades o deficiencias persistentes que pueden impedir a las personas gobernarse a sí mismas, y por tanto de acuerdo con el artículo 200 del Código Civil ser causa de incapacitación civil. Ello es admisible en casos muy evolucionados de dependencias graves, sin embargo es difícil en otras situaciones, en las que únicamente se afecta la capacidad en determinados momentos, y por tanto al no ser persistentes, no puede establecerse la incapacidad de autogobierno. En estos casos podría plantearse la valoración de una incapacitación parcial, en relación con el manejo de los bienes, de hecho sí puede relacionarse la drogodependencia con la prodigalidad, puesto que es frecuente que el adicto, con el fin de satisfacer su necesidad desvíe todos sus recursos a este fin, poniendo en peligro el sustento familiar o incluso generando un gravamen insoportable para los recursos del núcleo familiar.

Por otra parte los actos jurídicos realizados bajo los efectos de una intoxicación plena son susceptibles de posterior nulidad, por estimar que en estos momentos el sujeto no poseía suficiente capacidad para dar consentimiento válido.

En relación con los internamientos involuntarios, en principio hay que decir que en general no debe recurrirse a ello, por cuanto la base de su tratamiento es la voluntariedad; sin embargo en circunstancias excepcionales podría recurrirse a ello sobre todo para protección de la propia integridad o vida del toxicómano o la protección de terceros.

\section{DROGASY DERECHO LABORAL}

Los problemas que plantean las drogas en el mundo laboral se relacionan con una menor aptitud para responder adecuadamente a las exigencias del puesto de trabajo. 
El Estatuto de los Trabajadores considera dentro de las causas de despido disciplinario la toxicomanía si repercute negativamente en el trabajo. Además pueden ser causa de la pérdida del derecho a indemnización tras un accidente laboral y de incapacidad temporal o permanente, lo que puede hacer necesaria la valoración médico legal.

Ferreras de la Fuente, Pérez Urdaniz y col (2001) propone que a los efectos de determinar la capacidad laboral en las dependencias se realice una valoración multiaxial siguiendo los criterios de la DSM IV y requiriéndose para el reconocimiento de una invalidez absoluta que en el eje I se reseñe otra sintomatología psiquiátrica, en el Eje II se haga constar la base genética o el trastorno de la personalidad, en el eje II se refiera patología orgánica, en el Eje IV se contemplen los problemas psicosociales derivados del abuso y en el Eje $V$ el nivel previo y actual de funcionamiento familiar, social y laboral.

\section{DROGASY DERECHO ADMINISTRATIVO}

En este ámbito son varias las disposiciones que hacen referencia a las drogodependencias por las que puede solicitarse una valoración médico legal, así:

-El R.D. 22/1985 de 4 de diciembre que regula los permisos de armas y conductores de vehículos, recoge entre las causas de denegación de licencias, permiso y tarjeta de armas, la dependencia a estupefacientes y psicotropos; y de la obtención o renovación del permiso o licencia de conducción las toxicodependencias.

-La Orden 52/1986 de 17 de junio sobre exclusiones para el ingreso en determinados cuerpos y escalas de las fuerzas armadas, excluye a los sujetos con evidencia de consumo de sustancias piscotropas sin afinidad terapéutica comprobada analíticamente.

- La orden 75/1989 de 2 de Octubre relativa al cuadro médico de exclusiones para el ingreso en centros docentes militares de grado superior, recoge entre las causas, el hábito a drogas.

-El Comité Olímpico Internacional, recoge en la lista de sustancias dopantes los estimulantes y narcóticos y como sustancias sometidas a ciertas restricciones, la marihuana o cáñamo.

-El RDL 339/1990 de 2 de Marzo, texto articulado de la ley de tráfico, circulación de vehículos a motor y seguridad vial modificado por Ley 5/ 1997 de 24 de marzo, en su artículo 65, recoge como infracciones muy graves la conducción bajo los efectos de estupefacientes, psicotrópicos y cualquier otra sustancia análoga.
-El RDL 13/1992 de 17 de Enero. Reglamento general de Circulación en su articulo 27, dice que no podrá circular por las vías objeto de la legislación sobre tráfico circulación de vehículos a motor y seguridad vial, el conductor que haya ingerido o incorporado a su organismo drogas tóxicas o estupefacientes o se encuentre bajo los efectos de medicamentos $u$ otras sustancias que alteren el estado físico o mental apropiado para hacerlo sin peligro.

-La OM 74/1992 de 14 de Octubre de valoración psicofísica de los pilotos militares, en sus artículos 338 y 339 excluyen a los que consumen sustancias psicotropas y drogas.

- Las normas para la determinación de la aptitud psicofísica para el servicio militar (RD 1107/1993 modificada parcialmente por el RD 1410/94) recoge que el consumo excesivo de alcohol y otros tóxicos puede generar una inaptitud para ciertos destinos que debe ser periódicamente revisada, y las drogodependencias una inaptitud absoluta si existen signos objetivos y es grave con significativa repercusión funcional.

-La OM de 14 de julio de 1995 sobre títulos y licencias aeronáuticos civiles, (pilotos civiles), dice que el solicitante no tendrá historia clínica comprobada ni diagnóstico clínico de dependencia a fármacos, ni de alcoholismo.

-El R.D. 190/1996 de 9 de febrero. Reglamento penitenciario, recoge en varios artículos la posibilidad de los drogodependientes de seguir programas de tratamiento y deshabituación.

\section{El informe pericial en materia de drogas.}

El informe pericial es formalmente un documento que materializa la respuesta a las cuestiones sobre las que se pregunta al perito. Debe elaborarse de forma concreta y precisa de forma que aporte a los juristas la información requerida de forma comprensible y adaptada a sus necesidades.

El informe pericial debe reunir unas características formales y otras materiales o de contenido.

Desde el punto de vista formal el informe pericial es un documento escrito y normalmente de una extensión considerable que se estructura en una serie de apartados: Preámbulo, parte expositiva, consideraciones médico-legales, conclusiones y fórmula final, que Micó (1996) agrupa en 3 categorías:

1.- Apartados de carácter administrativo, como son: 1) la fórmula inicial que permite la identificación del perito, el solicitante y el objeto de la prueba. 2) Los datos en los que se basa el informe o fuentes de información de las que dispone el perito y 3) Fórmula final, cuyo dato 
más relevante suele ser la fecha de emisión del informe.

2.- La parte expositiva del informe o de carácter médico-psiquiátrico, en la que se recogen las actuaciones del perito y los datos obtenidos de las mismas.

3.- Apartados de carácter médico forense o psiquiátrico forense: 1) Las consideraciones medico-legales, que supone una valoración de los datos obtenidos atendiendo las cuestiones planteadas y 2) Las conclusiones, que deben suponer una respuesta razonada a las cuestiones.

En su redacción debe procurar el perito ser claro en la exposición, evitar los tecnicismos y los términos confusos, y si son imprescindibles debe aclararlos, debe razonar sus conclusiones y debe orientarlo a responder las cuestiones jurídicas que se le plantean.

Desde el punto de vista material en un informe pericial en relación con el consumo de drogas cualquiera que sea el ámbito del derecho deben valorarse los siguientes aspectos (Carrasco y Maza, 1996):

1.- La acreditación o no de la existencia de trastornos derivados del consumo de sustancias.

2.- La valoración del trastorno en cuanto a los efectos psicopatológicos generales y su repercusión jurídica, tanto en el momento de los hechos como en el momento de la exploración.

3.- Valoración global de la personalidad y existencia de otras patologias asociadas sistémicas o psiquiátricas.

4.- Pronóstico y actitud frente al problema, así como los tratamientos que esté realizando o intentos terapéuticos.

\section{La acreditación o no de la existencia de trastor- nos derivados del consumo o inducidos por sus- tancias.}

Se basara en el diagnostico clínico que se fundamenta básicamente en la entrevista y la exploración física.

Rubio y Rosenberg (2001) proponen como instrumentos para evaluar los trastornos por el uso de sustancias la utilización de 3 tipos de instrumentos:

a) Instrumentos de detección o cribaje: Suelen ser escalas de pocas preguntas entre las que refieren la Escala de dependencia del alcohol (ADS: Alcoholism Dependence Scale), la escala de valoración del estilo de vida de Dartmouth (DALI: Dartmouth Assessment of Lifestyle Instrument), la escala de alcoholismo de MacAndrew (MAC: MacAndrew Alcoholism Scale) etc. b) Entrevistas diagnósticas: Suele tratarse de entrevistas estructuras adaptadas a las diferentes clasificaciones y de más utilidad en proyectos de investigación que en la práctica clínica, entre las que refieren la diagnostic interview schedule (DIS) por la que se intentaba establecer diferencias entre trastornos inducidos y no inducidos, la AUDADIS para la valoración de trastornos por uso de alcohol y discapacidades asociadas, la PRISM (Psychiatric reseca interview for sustance and mental disorders) para la investigación psiquiátrica que discrimine los trastornos psiquiátricos de los inducidos por el uso de drogas, etc..

También son de utilidad otras escalas que evalúan trastornos psicopatológicos (escalas de depresión y de ansiedad), las escalas para la evaluación de la gravedad de la dependencia, y los marcadores biológicos.

c) Escalas clínicas o instrumentos para cuidadores: Están basadas en la información obtenida durante un periodo de tiempo tras la observación directa por familiares, cuidadores y el autoinforme. Dentro de estas escalas ha dado muy buenos resultados la propuesta por Barry y cols en 1995, pues a su fácil aplicación y a sus excelentes cualidades psicométricas, las preguntas tienen una adecuada concordancia con la entrevista estructurada acorde al DSM.

\section{La valoración del trastorno en cuanto a los efec- tos psicopatológicos generales y su repercusión jurídica, tanto en el momento de los hechos como en el momento de la exploración.}

Se trata de hacer una aproximación al estado del sujeto en el momento de los hechos en relación con dos criterios:

1. Criterio psicopatológico: Determinación de la situación médica del paciente atendiendo a las 3 situaciones más paradigmáticas:

- Intoxicación aguda.

- Situación de consumo crónico (Adicción), y de la patología inducida por el consumo.

- Situación clínica de síndrome de abstinencia.

La valoración de la situación médica debe hacerse atendiendo a un triple criterio: a) Cualitativo: naturaleza de los síntomas o signos clínicos. c) Cuantitativo: Gravedad del cuadro clínico, y c) Cronológico: momento de aparición de los síntomas en relación con la administración de la droga o el momento de su supresión.

2. Un criterio médico-legal: Relación entre el estado psíquico del paciente y los hechos, lo que supone: 
2.1. Un análisis estructural de su funciones cognitivas y volitivas.

De una forma general podemos decir que los drogadictos tienen mermada su voluntad en el sentido de que tienen una aversión patológica el síndrome de abstinencia y una afectación motivacional durante el consumo crónico que incluye la angustia premonitoria ante la carencia de sustancia, siendo en general menor la afectación de la esfera cognitiva.

El sujeto adicto sabe y conoce los efectos de las drogas y de su falta pero también conoce la posibilidad de acudir a un centro asistencial para atenuar los efectos del síndrome de abstinencia e incluso iniciar una deshabituación, por tanto no puede decirse que el drogadicto esta sometido a una fatalidad delictiva según un modelo determinista sino que existe un margen opcional para evitar la conducta delictiva. Por ello la jurisprudencia ha alcanzado un cierto consenso en considerar a los drogadictos como imputables de los delitos, salvo que pueda demostrarse taxativamente alguna de las situaciones clínicas paradigmáticas, o que debido al consumo crónico o a la concurrencia de circunstancias individuales el paciente presente un cuadro de psicosis tóxica, hoy denominados trastornos orgánicos inducidos por sustancias.

2.2. Valorar la gravedad de la toxicomanía, teniendo en cuenta en primer lugar el tiempo transcurrido entre el hecho delictivo/ detención y el reconocimiento médico, y basándonos en los siguientes factores:

a) Estudio de los antecedentes del paciente en los que figuraran los tratamiento de desintoxicación o bien la asistencia.

b) La aparición de un síndrome de abstinencia después de la detención

c) Existencia de signos de intoxicación o abstinencia durante el reconocimiento.

d) Existencia de estigmas físicos: afectación del estado general (delgadez extrema, apatía, palidez,...), esclerosis venosa, enfermedades infecciosas relacionadas con la toxicomanía, etc.

e) Fracaso en la vida socio-laboral y familiar

f) Estudio de la personalidad y/o detección de una enfermedad mental subyacente.

g) Estudio toxicológico para la determinación analítica de la droga

\section{Valoración global de la personalidad y existencia de otras patologías asociadas sistémicas o psi- quiátricas.}

Existe una gran polémica sobre si existe un perfil típico de personalidad del drogodependiente o personalidad toxicómana, y aunque la doctrina mayoritaria opina que no, si se admite que de forma general la personalidad del consumidor de drogas se estructura sobre una alteración afectiva, mezcla de ansiedad, depresión y baja autoestima, y que la personalidad influye en la motivación que conduce al consumo, siendo válida la afirmación que en 1965 hizo Jaffe': el neurótico puede utilizar la droga para aliviar la ansiedad (euforia negativa), el psicópata para conseguir una emoción (euforia positiva) y el psicótico, para aliviar la tensión o suprimir las ideas delirantes.

La coexistencia del consumo de drogas con otra patología psiquiátrica, la denominada patología dual, supone un incremento de la complejidad en la valoración, pues es bien conocido que esta asociación no sólo ensombrece el pronóstico del los trastornos relacionados con el consumo de sustancias (trastornos inducidos), sino que el uso de estas influye negativamente en la patología psiquiátrica subyacente, por tanto resulta imprescindible:

1. La realización de un correcto diagnóstico diferencial entre el trastorno inducido y el trastorno independiente.

2. La evaluación de la influencia del consumo de drogas sobre otros trastornos psiquiátricos, pues como claramente establece Dixon (1999), esta asociación suele traducirse en un incremento de las conductas agresivas o violentas, en un incumplimiento del plan terapéutico, en la exacerbación de los síntomas psicóticos, en recaídas en la enfermedad psiquiátrica y en un mayor coste asistencial.

Aún siendo muchas las dificultades metodológicas para el correcto diagnóstico, el seguimiento de los criterios establecidos en las modernas clasificaciones de las enfermedades mentales (DSM IV o CIE X) nos han facilitado la estandarización y la fiabilidad diagnóstica, y el desarrollo de numerosos trabajos en el campo de la patología dual (Rubio, López-Muñoz, y col, 2001) nos ha permitido establecer unos criterios básico que puedan servirnos de orientación, que en un breve resumen exponemos a continuación:

1.- Diagnóstico diferencial entre el trastorno inducido y el independiente. Rubio y Nunes (2001) nos proponen una actuación en 3 pasos:

Primer paso: Establecer una relación entre los síntomas psiquiátricos y el consumo de drogas. Si no

Citado por Delgado. 
existe el diagnostico se orientara hacia una trastorno independiente, por el contrario si observamos relación debemos seguir observando, para explorar las características psicopatológicas y la cronología de los trastornos.

Segundo paso: Debemos determinas si los trastornos que presenta son más graves de los que suelen aparecer durante los episodios de intoxicación o de síndrome de abstinencia. Si se corresponden clínica y cronológicamente podemos diagnosticarlos como intoxicación o síndrome de abstinencia, y si son más graves o falta la correspondencia deberemos continuar las exploraciones.

Tercer paso: Se realizar un estudio cronológico de la aparición de los trastornos, de los antecedentes personales y familiares y observar si se resuelve el episodio tras un tiempo de abstinencia.

En resumen nos hace pensar que no se trata de un trastorno inducido por drogas los siguientes datos: a) el comienzo del trastorno psiquiátrico antes que el consumo regular de drogas; b) las diferencias en intensidad, frecuencia y tipo de problemas, siendo muy frecuente que los trastornos inducidos se acompañen de alteraciones del nivel de conciencia y que su remisión es paralela a la eliminación del tóxico; c) La persistencia del trastorno tras varias semanas de abstinencia; d) la existencia de antecedentes familiares con el mismo trastorno y e) el fracaso terapéutico tanto de su conducta adictiva como de otros trastornos similares.

2.- Evaluación de la influencia del consumo de drogas sobre otros trastornos psiquiátricos.

Según Rubio y Rosenberg (2001) la evaluación puede hacerse recurriendo a medios prospectivos, retrospectivos y objetivos.

Los métodos prospectivos son una variante de la monitorización y por tanto una alternativa a la observación directa.

Los métodos retrospectivos se basan en autoinformes referidos a un periodo de tiempo, dependiendo su exactitud de 4 clases de variables: a) Las características del paciente (sobriedad, deseabilidad social de la respuesta); b) Factores que aumentan o disminuyen la exactitud de la respuesta (empatía con el terapeuta, confidencialidad de la entrevista, momento de la entrevista, etc.); c) Factores motivacionales relacionados con lo que espera obtener; y d) Capacidad cognitiva, pues el deterioro de la atención, la comprensión verbal, o de otras funciones, pueden influir en la exactitud de las respuestas.

Los métodos objetivos incluyen los análisis de orina, sangre, aire espirado y pruebas de laboratorio que pueden orientar sobre el consumo reciente, sobre las consecuencias del consumo crónico de una sustancia, etc.

\section{Pronóstico y actitud frente al problema, así como los tratamientos que esté realizando o intentos terapéuticos.}

En la valoración del pronóstico del drogodependiente, tanto desde la peligrosidad criminal como del pronóstico medico, teniendo en cuenta que desde este punto de vista es una enfermedad crónica y con tendencia a la recaída, será muy importante tener en cuenta una serie de elementos que de alguna manera condicionaran el éxito o el fracaso de la asistencia medica o psicosocial al problema:

4.1. La motivación para el consumo.

4.2. La motivación para abandonar el consumo.

4.3. El Plan terapéutico.

\subsection{Motivación para el consumo.}

Independientemente del factor que induce a una persona a probar las drogas, como dice Laurie(1973) deben darse 3 factores para que se de el peligro de toxicomanía: 1) Una insuficiencia psicológica que predisponga al consumo de estas sustancias. 2) Una crisis o conflicto inopinado y 3) Una proposición o invitación al consumo en ese determinado momento.

\subsection{La motivación para abandonar el consumo.}

Teniendo en cuenta que rara vez el adicto toma conciencia de su situación, los motivos y circunstancian que le llevan a la solicitud de ayuda médica y a la aceptación de someterse a un tratamiento de desintoxicación o deshabituación suelen ser tal como exponen Seva (1979) y González Allepuz (1999): 1) la aparición de los efectos patológicos orgánicos de la droga; 2) las alteraciones psíquicas inducidas por las drogas o los derivados del consumo; 3)el caos psicosocial que ésta le ha procurado (problemas familiares o laborales fundamentalmente); y 4) la presencia de una situación conflictiva, incluso delictiva, que tiene consecuencias legales.

\subsection{El Plan terapéutico.}

En la asistencia a un drogodependiente deben plantearse objetivos variables que intenten llegar a la abstinencia absoluta y la plena integración social, pero sin olvidar que en muchas ocasiones sólo se consiguen objetivos intermedios pues como dicen Gonzalez y Rubio (2001) la drogodependencia es una enfermedad crónica con recidivas que se inicia con el primer consumo gratificante y está expuesta a recaídas motivadas por factores individuales y del medio.

Desde esta perspectiva una correcta actuación requiere un planteamiento de objetivos con un orden 
de prelación: 1) Conseguir la abstinencia lo más prolongada posible, siendo lo ideal de por vida; 2) Identificar el trastorno psiquiátrico que genero la conducta o los trastornos inducidos por el consumo para implantar el tratamiento adecuado; 3) Diagnosticar y tratar las patologías orgánicas relacionadas con el consumo; 4) Modificar los hábito de consumo para reducir los riesgos derivados de la forma de uso, intentar disminuir o sustituir la sustancia etc., 5) Disminuir el proselitismo; 6) Aliviar la carga policial, judicial y penal y 7) Mejorar la actitud de aceptación social de su drogodependencia para evitar la marginación que perpetúe los hábitos.

Por ello un Plan terapéutico integral debe realizarse en 4 fases: 1) Acogida: para evaluar la demanda y el entorno del paciente para establecer el programa personal específico; 2) Desintoxicación: que puede realizarse por extinción lenta o brusca y de forma ambulatoria y hospitalaria en dependencia de los apoyos, y la situación orgánica y psíquica del paciente; 3) Rehabilitación o Deshabituación: Es un proceso terapéutico de duración variable pero en general largo durante el cual el paciente restablece su estado psicofísico y aprende a abordar los conflictos para conseguir una cultura de rechazo a la droga que le propicie un cambio en el estilo de vida; y 4) Reinserción: la nueva adaptación al medio familiar, social y laboral.

En definitiva un informe pericial en materia de consumo de drogas debe dejar constancia del mayor número de datos posibles y de las fuentes de información de las que ha dispuesto el perito puesto que el grado de fiabilidad que puede merecer un informe pericial vendrá muy ligado a los elementos y datos que el perito seleccione para emitir su opinión técnica, y que tal como han dejado de manifiesto diversas sentencias la escasez de elementos induce a dudar del resultado. En este sentido no debemos olvidar que si bien el dictamen pericial no es vinculante para el juez, éste para la correcta apreciación de la prueba debe implicarse activamente en su análisis, esforzándose para comprender el planteamiento técnico aducido no quedando su actuación reducida a la estimación o desestimación sino a la motivación de la misma, y que sigue siendo valida la afirmación hecha por A. Paré "Los jueces deciden según se les informa".

\section{BIBLIOGRAFIA}

Arechederra, J.J, y Arias, F (2000): Drogodependencias y Psiquiatría: Aspectos clínicos y legales. You \& Us. Madrid.

Cabrera, J y Fuertes, J.C.: La enfermedad mental ante la Ley. ELA. Madrid 1994.

Carrasco, J.J. y Maza, J.M (1996): Psiquiatría legal y Forense. Ley-actualidad, S.A.; Madrid.
Código Civil. Tecnos. Madrid 1996

Código Penal. Tirant lo Blanch, Valencia 1995

Christiani, E y Stübing, P (1977): Abuso y dependencia de las drogas. Deutcher Arzteverlag.

Delgado., S (1994): Psiquiatría Legal y Forense. Colex. Madrid

Delgado, S: El drogodependiente ante los Tribunales de Justicia. Colex. Madrid, 1998.

Dixon, L (1999): Dual diagnosis of substance abuse in schizophrenia: prevalence and impact on outcomes. Schizophr. Res 35: S93-S100.

DSM IV, (1995): Manual diagnóstico y estadístico de los trastornos mentales. Masson, Barcelona.

Ferreras, A. ;Pérez Urdaniz, A. ; Molina, R; Solana, et al (2001): Manual de valoración de la Incapacidad Laboral en las enfermedades Psiquiátricas. INMST. Madrid. pag 72.

Gisbert., J.A (1991): Medicina Legal y Toxicología, $4^{a}$ edic. Salvat. Barcelona. 1991.

Gonzalez Allepuz, J (1999): La desintoxicación. En Seva, A. y cols. La drogadicción. Edit. CAl. Zaragoza.

Gonzalez Allepuz, J y Rubio Larrosa, V (2001): Las toxicomanías. En Seva, A: Tratado de psiquiatría. INO. Zaragoza.

Gracia, L; Boldova, M.A. y Alastuey, C (1998): Lecciones de consecuencias jurídicas del delito. Tirant lo Blanch, Valencia.

Jaffe: Citado por Delgado S. (1998): En El drogodependiente ante los Tribunales de Justicia. Colex. Madrid.

Laurie, P (1973): Las drogas. Alianza Editorial. Madrid.

Ley 16/1970, de 4 de Agosto de Peligrosidad y Rehabilitación social.

Marcó, J; Martí, J.L y Pons, R (1990): Psiquiatría forense. Salvat. Barcelona.

Micó, F (1996): Psiquiatría forense en esquemas. Colex, Madrid.

Maurer, DW y Vogel, VH (1967): Narcotics and narcotic

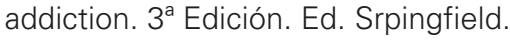

Ortega Monasterio, L (1991): Psicopatología Jurídica y Forense. PPU, Barcelona.

Reichel, H, citado por Ibáñez Guerra, E (1994): Personalidad y Delincuencia. En Cuadernos de Derecho Judicial: Aportaciones de la psicología al ámbito jurídico (Tomo XIX). CGPJ. Madrid. pp 129-166.

Rodes, F y Martí, J.B. (1997): Valoración médico legal del enfermo mental. Universidad de Alicante. Alicante.

Rubio, G y Nunes, E.V (2001): Instrumentos diagnósticos de trastornos psiquiátricos en poblaciones con abuso de sustancias (Capitulo 9: 191-200). En: Rubio, G, LópezMuñoz, F, Álamo, C y Santo-Domingo, J: Trastornos psiquiátricos y abuso de sustancias. Edit Panamericana., Madrid.

Rubio, G y Rosenberg, S.D. (2001): Evaluación del uso de drogas y de los trastornos por uso de sustancias en sujetos con otros trastornos psiquiátricos (Capitulo 10:201-219). En: Rubio, G, López-Muñoz, F, Álamo, C y Santo-Domingo, J: Trastornos psiquiátricos y abuso de sustancias. Edit Panamericana., Madrid.

San Martín, H (1982): La crisis mundial de la salud. Problemas actuales de epidemiología social. Karpos. Madrid. 
Serrat, D. y Morales, C.(1999): Los problemas legales que pueden plantear el consumo de drogas. En: Seva, A. y cols. La drogadicción. CAl. Zaragoza.
Seva, A (1979): Psiquiatría clínica. Espaxs. Barcelona. Seva, A (2001): Tratado de psiquiatría. INO. Zaragoza. 
